\title{
Recyclable nanocellulose-confined palladium nanoparticles with enhanced room-temperature catalytic activity and chemoselectivity
}

\author{
Juan Meng ${ }^{\dagger}$, Yongzhuang $\mathrm{Liu}^{\dagger}$, Xiaochao Shi, Wenshuai Chen, Xianquan Zhang ${ }^{*}$ and Haipeng Yu*
}

\begin{abstract}
We describe the synthesis of even-dispersed palladium nanoparticles (Pd NPs) confined within a cellulose nanofiber (CNF) matrix for developing a high-performance and recyclable catalyst. The CNF matrix was composed of CNF-assembled mesoporous nanosheets and appeared as soft and hydrophilic foam. Ultrafine Pd NPs $(\sim 6 \mathrm{~nm})$ with highloading $(9.6 \mathrm{wt} \%)$ were in situ grown on these mesoporous nanosheets, and their dense spatial distributions were likely to generate nano-confinement catalytic effects on the reactants. Consequently, the CNF-confined Pd NPs (CNF-Pd) exhibited an enhanced room-temperature catalytic activity on the model reaction of 4-nitrophenol hydrogenation with a highest rate constant of $8.8 \times 10^{-3} \mathrm{~s}^{-1}$ and turnover frequency of $2640 \mathrm{~h}^{-1}$. The CNF-Pd catalyst possessed good chemical stability and recyclability in aqueous media which could be reused for at least six cycles without losing activity. Moreover, chemoselective reduction of 3-nitrostyrene was achieved with high yield $(80 \%-98 \%)$ of 3 -aminostyrene in alcohol/water cosolvent. Overall, this work demonstrates a positive nanoconfinement effect of CNFs for developing stable and recyclable metal NP catalysts.
\end{abstract}

Keywords: nanocellulose, deep eutectic solvents, palladium nanoparticles, catalysts, nano-confinement

\section{INTRODUCTION}

Metal nanoparticles (NPs) as heterogeneous catalysts are used in a wide range of applications such as organic synthesis [1], biorefinery [2], electrocatalysis [3,4], and environmental protection [5]. In particular, palladium (Pd) NPs have been widely investigated in cross-coupling [6], oxidation [7], decarbonylation [8], hydrogenation [9] and related reactions $[10,11]$. However, Pd NPs are generally unstable due to their large active surface areas and suffer from self-aggregation problems, which impair their properties and limit their catalytic applications [12-14].

In order to solve the above problems, various strategies have been attempted to stabilize Pd NPs including confinement of them in solids, polymers or ligands [15-18]. For example, carbon materials, including graphene [19], carbon nanotubes [20] and carbon fibers [21], have been used to load Pd NPs, and these carbon-loaded Pd NPs exhibit high catalytic turnover frequency (TOF) values of 1068-2520 h ${ }^{-1}$. Porous nanomaterials such as molecular sieves and metal-organic frameworks have also been used to anchor Pd NPs, and the Pd NPs confined within their nanopores exhibit excellent chemoselectivity of $99 \%$ at $353 \mathrm{~K}$ [22]. Recently, low-cost, nontoxic and biodegradable matters including chitosan, cellulose, and wood, have been used as natural templates for the syntheses of Pd NPs [23-26]. They are featured as having characteristics of renewability, sustainability, flexibility, hydrophilicity, biocompatibility, and easy processability. The combination of natural polymers and Pd NPs is highly expected to show unique advantages in catalytic reactions $[27,28]$. Nevertheless, the development of natural nanomaterials as novel subtle matrices for enhancing the catalytic activity and recyclability of Pd NPs is still in progress.

Nanocellulose has drawn broad attention due to its fine morphology, huge specific surface area, abundant active reaction groups, and decent stability both in water and most organic solvents [29]. The functionalized nanocellulose can be sustainable building blocks for constructing nanoscale matrices based on bottom-up assembly strategy to load Pd NPs [30]. For instance, cellulose nanocrystals (CNCs) were used as support materials of Pd NPs for catalytic hydrogenation at $293 \mathrm{~K}$ and

Key Laboratory of Bio-based Material Science and Technology of Ministry of Education, Northeast Forestry University, Harbin 150040, China

${ }^{\dagger}$ These authors contributed equally to this work.

* Corresponding authors (emails: yuhaipeng20000@nefu.edu.cn (Yu H); zhangxianquan2013@aliyun.com (Zhang X)) 
Heck coupling at 353-403 K [31-34]. In addition, 2,2,6,6tetramethyl-piperidin-1-oxyl (TEMPO)-oxidized cellulose nanofibers $(\mathrm{CNFs})$ were adopted as Pd carriers for using in discoloration of dyes and Suzuki reactions $[35,36]$. Although these attempts have made progress in the past, little attention has been laid on the spatial configuration of the as-prepared products, and the regulation of dense, even distribution of Pd NPs in CNFassembled matrix and the resultant nano-confinement effect have barely caught attention. Thus, the catalytic route is usually rigid and restricted, and the roomtemperature catalytic activity and chemoselectivity have not reached an ideal level.

In this study, a two-step synthesis method was proposed to fabricate high-loading and even-distributed Pd NPs with the CNF-assembled matrix as a chemical and spatial-confinement template (Scheme 1). A hydrated deep eutectic solvent (DES) combined with ultrasonic treatment was used to replace TEMPO to prepare carboxylic CNFs. The obtained carboxylic CNFs had high aspect ratios and rich carboxyl moieties, which provide a great possibility for them as carriers for in situ synthesis of Pd NPs. A three-dimensional (3D) hybrid foam of CNF with confined Pd NPs (CNF-Pd) was formed by simply freeze-drying the mixture of carboxylic CNFs and Pd NPs. The CNF-Pd hybrid foam was mainly composed of CNF-assembled mesoporous nanosheets, with evendistributed Pd NPs anchored on their surfaces. The nanoconfinement effect, catalytic activity and recyclability of the CNF-Pd foam as a catalyst were investigated by the hydrogenation reaction of model compound 4-nitrophenol at room temperature. The chemoselectivity performance of the CNF-Pd catalyst was also studied in the hydrogenation of 3-nitrostyrene (NS) to 3-aminostyrene (AS) or 3-nitroethylbenzene (ENB).

\section{EXPERIMENTAL SECTION}

\section{Preparation of carboxylic CNFs}

Choline chloride and oxalic acid dihydrate were mixed with a molar ratio of $1: 1$ at $80^{\circ} \mathrm{C}$ for $1 \mathrm{~h}$ to give a DES [37]. Cellulose pulp ( $0.5 \mathrm{~g})$ was mixed with the DES ( $10 \mathrm{~g})$ and water $(10 \mathrm{~g})$ in a Teflon-lined polyetheretherketone tank. The tank was heated at $110^{\circ} \mathrm{C}$ for $2 \mathrm{~h}$ to obtain carboxylic cellulose. The carboxylic cellulose was then diluted with water to a concentration of $0.4 \% \mathrm{w} / v$. The mixture was then treated using a JY99-IIDN ultrasonicator $(20 \mathrm{kHz}$, Scientz Biotechnology Co., Ningbo, China) at $800 \mathrm{~W}$ for $15 \mathrm{~min}$, resulting in $0.4 \% \mathrm{w} / \mathrm{v}$ carboxylic CNF suspension.

\section{Synthesis of CNF-Pd}

$\mathrm{PdCl}_{2}(17.7 \mathrm{mg})$ was dissolved in $\mathrm{HCl}\left(1 \mathrm{mmol} \mathrm{L}{ }^{-1}\right.$, $50 \mathrm{~mL}$ ) at room temperature with a magnetic stirrer. The above solution $(10 \mathrm{~mL})$ was added into the CNF suspension $(20 \mathrm{~mL}, 0.4 \mathrm{wt} \%)$ under vigorous stirring. An aqueous solution of $\mathrm{NaBH}_{4}(10 \mathrm{mg}, 1 \mathrm{~mL})$ was added dropwise to the mixture which then reacted at $4^{\circ} \mathrm{C}$ for $4 \mathrm{~h}$. The $\mathrm{Cl}^{-}$and unbound Pd NPs were removed by dialysis with deionized (DI) water by using regenerated cellulose membranes (molecular weight cutoff: 12$14 \mathrm{kDa}$, Spectrum Laboratories Inc., Rancho Dominguez, CA, USA). After dialysis, the product was freeze-dried to CNF-Pd foam for use.

\section{Characterizations}

The carboxyl group $(-\mathrm{COOH})$ content of CNFs was measured by conductometric titration with a DDS-11A conductivity meter (LEICI Instrument, Shanghai, China). Fourier transform infrared (FTIR) spectra were recorded over the absorbance range from 500 to $4000 \mathrm{~cm}^{-1}$ by using a Nicolet Magna 560 FTIR instrument (Thermo Fisher Scientific Inc., Waltham, USA). The Pd content in the CNF-Pd was determined by inductively coupled plasma spectrometry (ICP, PE Optima8300, USA). The samples were digested using nitric acid/perchloric acid $(5: 1, v / v)$ with high-temperature sterilization at $200^{\circ} \mathrm{C}$ for $6 \mathrm{~h}$. The resulting solutions were diluted to $10 \mathrm{ppb}$ level for ICP analysis. X-ray photoelectron spectroscopy (XPS) analysis was performed by using a $\mathrm{K} \alpha$ system (Thermo

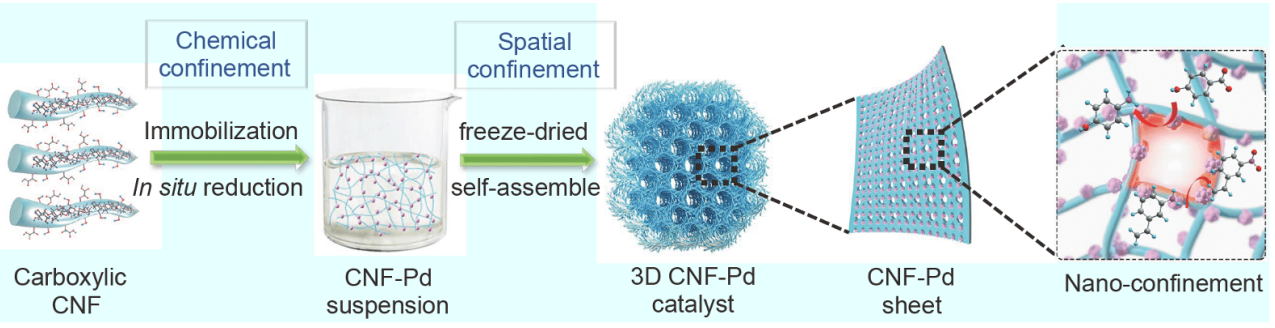

Scheme 1 Schematic illustration of a two-step confinement strategy for preparing the CNF-Pd catalyst. 
Fisher Scientific, Basingstoke, West Sussex, UK) operated at $14 \mathrm{kV}$. Nitrogen sorption analysis was performed by using a 3H-2000PS2 Brunauer-Emmett-Teller (BET) surface area analyser (Beishide ST Ltd., Beijing, China). Specific surface area was calculated with the BET method. Pore volume and pore-size distribution were calculated by using the Barrett-Joyner-Halenda model based on the adsorption branches of the isotherms. X-ray diffraction (XRD) was performed on a $\mathrm{D} / \max 2200$ diffractometer (Rigaku, Japan), with $\mathrm{Ni}$-filtered $\mathrm{Cu} \mathrm{Ka}$ radiation at $40 \mathrm{kV}$ and $30 \mathrm{~mA}$. The diffraction data were collected in the range of $2 \theta=10^{\circ}-90^{\circ}$ at a scanning rate of $2^{\circ} \mathrm{min}^{-1}$. The Pd crystallite dimensions were calculated based on the Scherrer equation. A Tecnai G2 transmission electron microscope (TEM; FEI, USA) operated at $100 \mathrm{kV}$ was used for TEM imaging. The CNFs were stained with phosphotungstic acid (1\%) to enhance the contrast before TEM imaging. The samples were prepared by depositing $10 \mu \mathrm{L}$ drop of $0.01 \%$ aqueous suspension on a carboncoated TEM grid (300-mesh copper, formvar-carbon, Ted Pella Inc., USA). The sizes of Pd NPs were measured from the TEM images by using a TDY-V5.2 image analysis system (Tianhong Precision Instruments, Beijing, China). Scanning electron microscopy (SEM) images of samples were taken by using a JSM-7500F microscope (JEOL, Japan) at $15 \mathrm{kV}$. Before observation, the samples were coated with platinum by using a vacuum sputter coater. Elemental analysis and elemental mapping at the microstructural level were performed by the energy-dispersive $\mathrm{X}$-ray spectrometry (EDS) attached to the SEM.

\section{Catalytic performance of CNF-Pd}

The room-temperature catalytic activity and recyclability of CNF-Pd were studied through catalytic hydrogenation of 4-nitrophenol to 4-aminophenol. Generally, 4nitrophenol (0.5 mmol, $70 \mathrm{mg})$, CNF-Pd (5 mg, $9.6 \mathrm{wt} \%$ Pd loading) and $\mathrm{NaBH}_{4}(2 \mathrm{mmol}, 76 \mathrm{mg})$ were added in $10 \mathrm{~mL}$ of water with continuous stirring at room temperature. Ultraviolet-visible (UV-Vis) spectrophotometer (Lambda 750, Perkin Elmer, USA) was used to reflect the changes. After the indicated reaction time (typically 300-900 s), the CNF-Pd was recycled from the mixture by centrifugation and directly reused for a next catalytic reaction after washing properly with water. The products in aqueous solution were extracted with ethyl acetate $(5 \mathrm{~mL} \times 3)$, and the combined organic phase was concentrated in vacuo and filtered for analysis. The products were identified by gas chromatography-mass spectroscopy (GC-MS, Agilent 7890A-7000B, HP5-MS capillary column). The yield and selectivity were quantified by UV and GC equipped with flame ionization detector (GCFID, Agilent 7890B GC, HP5-MS capillary column). The temperature program consisted of $10^{\circ} \mathrm{C} \mathrm{min}^{-1}$ heating steps from 80 to $280^{\circ} \mathrm{C}$.

The kinetic reaction rate constant $(k)$ was calculated by Equation (1).

$\ln \left(C_{t} / C_{0}\right)=-k t$,

where $C_{0}$ and $C_{t}$ correspond to the concentrations of nitrophenol at initial time and reaction time $t$.

The TOF value was calculated based on Equation (2).

$\mathrm{TOF}=\frac{\text { mole of converted substrate }}{\text { mole catalyst } \times \text { reaction time }}$.

Catalytic hydrogenation of NS was used to verify the chemoselectivity of CNF-Pd combined with different solvents. Generally, NS $(0.5 \mathrm{mmol}, 75 \mathrm{mg})$, CNF-Pd (5 mg, $9.6 \mathrm{wt} \% \mathrm{Pd}$ loading) and $\mathrm{NaBH}_{4}(2 \mathrm{mmol}, 76 \mathrm{mg}$ ) were added in $10 \mathrm{~mL}$ of water or alcohol/water co-solvent with continuous stirring at room temperature. After the indicated reaction time (typically 1-3 h), the products were separated from CNF-Pd by centrifugation and concentrated in vacuo. Ethyl acetate $(5 \mathrm{~mL} \times 3)$ was used for extraction and the combined organic phase was concentrated in vacuo and filtered for analysis. The products were identified and quantified by GC-MS and GCFID as previously described.

\section{RESULTS AND DISCUSSION}

The carboxyl content of CNFs should first be concerned because they are responsible for the confinement of dense and uniform Pd NPs on their surfaces. More carboxyl groups on CNFs can provide more active sites for anchoring $\mathrm{Pd}^{2+}$ ions. The DES pretreatment is considered as an environmentally friendly method for both the preparation and the carboxylation of CNFs [37-40]. Here, a $50 \%$ hydrated choline chloride/oxalic acid dihydrate DES was used to pretreat the cellulose to produce carboxylic CNFs. As listed in Table 1, the carboxyl content increased with the reaction time, but the yield of CNFs decreased in the meantime. Therefore, a reaction time of $4 \mathrm{~h}$ seemed too long for the pretreatment, because the CNF yield dropped significantly to $58 \%$ due to severe degradation during the process. Considering both the yield and the carboxyl content, a reaction time of $2 \mathrm{~h}$ was deemed optimal. The hydrated DES pretreatment also promoted the ultrasonic nanofibration of CNFs, and homogeneous CNF suspension was obtained as shown in Fig. S1. The diameters of CNFs mainly concentrated in 5-10 nm, and the aspect ratios were as high as several hundred (Fig. S2). The presence of FTIR absorption peak at $1725 \mathrm{~cm}^{-1}$ in- 
Table 1 Yields and carboxyl contents of CNFs after various reaction times in hydrated DES

\begin{tabular}{cccc}
\hline Sample & Reaction time (h) & Yield (wt\%) & $\begin{array}{c}-\mathrm{COOH} \\
\left(\mathrm{mmol} \mathrm{g}^{-1}\right)\end{array}$ \\
\hline \multirow{4}{*}{ CNFs } & 0.5 & 99.98 & 0.068 \\
& 1.0 & 87.51 & 0.113 \\
& 2.0 & 80.62 & 0.164 \\
& 4.0 & 58.00 & 0.213 \\
\hline
\end{tabular}

dicated the existence of $\mathrm{C}=\mathrm{O}$ due to the DES pretreatment (Fig. S3). Other absorption peaks at 1637, 1424, $1373,1108,1027$ and $890 \mathrm{~cm}^{-1}$ agreed well with those of pure cellulose.

The CNF-Pd was synthesized through a two-step confinement process (Scheme 1). During the process, UV-Vis spectra were recorded to reflect the chemical crosslinking reactions (Fig. 1a). The $\mathrm{PdCl}_{2}$ solution originally showed typical absorption peaks of $\mathrm{Pd}^{2+}$ at 208 and $233 \mathrm{~nm}$. When $\mathrm{Pd}^{2+}$ was chemically crosslinked with the carboxyl groups of CNFs, their homogeneous CNF-Pd suspension still showed typical absorption peaks as those of $\mathrm{PdCl}_{2}$ solution. After the in situ reduction by adding $\mathrm{NaBH}_{4}$, the color of the mixture solution changed from light yellow to black (Fig. 1a inset), and typical $\mathrm{Pd}^{2+}$ absorption peaks disappeared from the UV-Vis spectrum. This was consistent with the reduction of $\operatorname{Pd}(\mathrm{II})$ to $\operatorname{Pd}(0)$ [41]. The CNF-Pd suspension was stable even after a deposition of six months (Fig. S4a).

When the CNF-Pd suspension was freeze-dried into CNF-Pd foam (Fig. S4b), its phase structure was detected by XRD (Fig. 1b). The diffraction peaks at $2 \theta=40.0^{\circ}$, $46.4^{\circ}, 68.0^{\circ}$, and $82.0^{\circ}$ in the curve of CNF-Pd corresponded to the (111), (200), (220), and (311) lattice planes of Pd (JCPDS\# 46-1043). Typical cellulose I peaks corresponding to the (110) and (200) lattice planes were also observed, suggesting the hydrated DES pretreatment and $\mathrm{NaBH}_{4}$ reduction process did not change the crystal type of cellulose.

The chemical bonding state between CNF and Pd was further investigated by XPS. The peaks of $\mathrm{C}, \mathrm{O}$ and $\mathrm{Pd}$ elements were found in the wide-scan spectra of CNF-Pd (Fig. 1c). From the high-resolution scan spectrum of $\mathrm{Pd}$ 3d (Fig. 1d), the $\operatorname{Pd}^{0} 3 \mathrm{~d}_{5 / 2}$ and $3 \mathrm{~d}_{3 / 2}$ peaks at 334.9 and $340.6 \mathrm{eV}$ could be identified clearly. Pd in the oxidation state (peaks at $336.8 \mathrm{eV}$ ) was also found, which was possibly due to the coordination of Pd NPs with carboxyl/
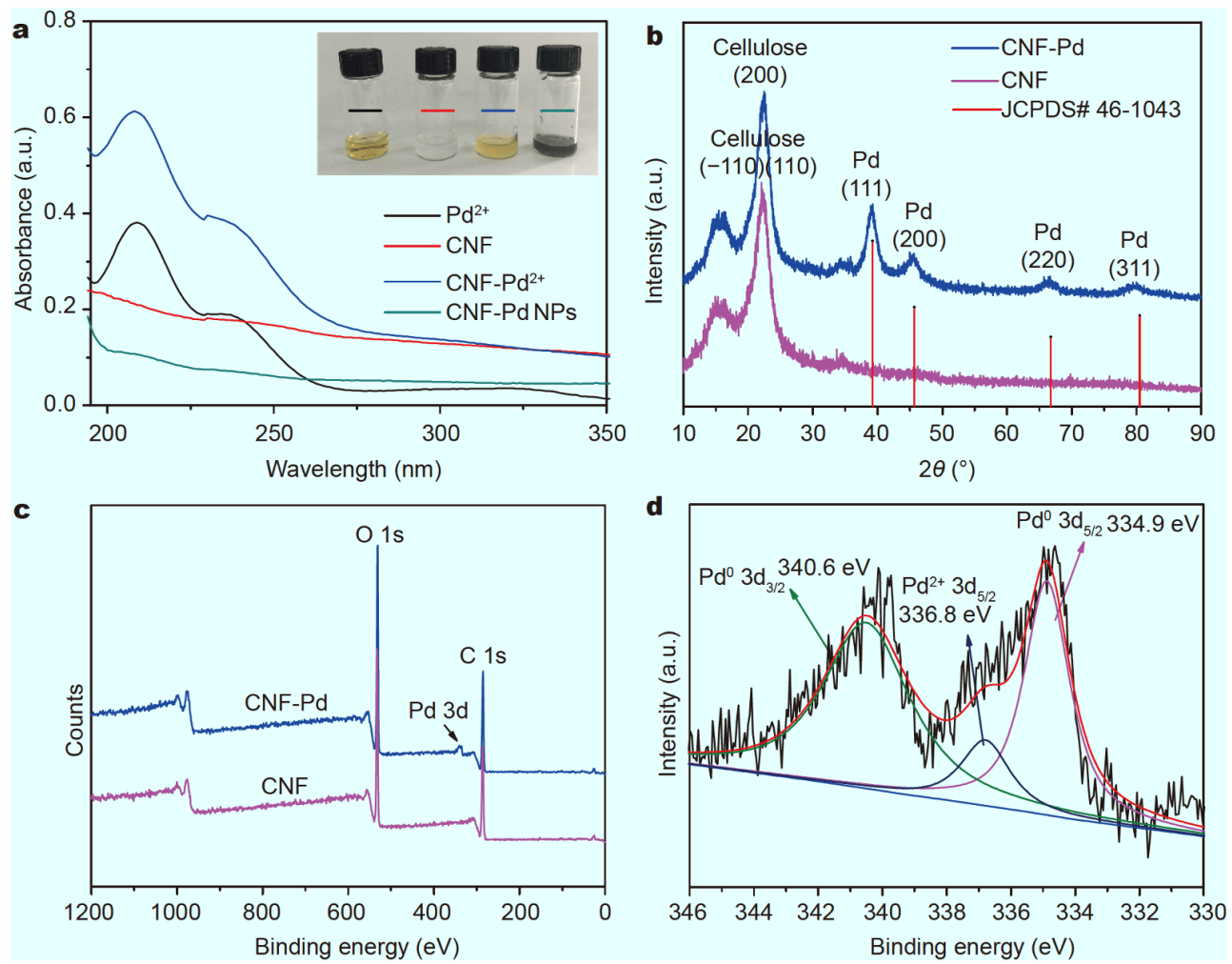

Figure 1 Physicochemical characterizations of the as-prepared CNF-Pd. (a) UV-Vis spectra and photos (inset) of $\mathrm{PdCl}{ }_{2}$, 0.4 wt $\% \mathrm{CNF}$ suspension, mixture solution of $0.4 \mathrm{wt} \% \mathrm{CNF}$ and $\mathrm{PdCl}_{2}$, and the resulting CNF-Pd suspension after adding $\mathrm{NaBH}_{4}$; (b) XRD spectra of $\mathrm{CNF}-\mathrm{Pd}$, CNF and $\mathrm{Pd}$ (JCPDS\# 46-1043); (c) XPS spectra of CNF-Pd and CNF; (d) the high-resolution scan spectra of Pd 3d. 
hydroxyl groups of CNFs or oxidation in the air [42-44]. The peaks of $\mathrm{C} 1 \mathrm{~s}$ at $284.8,286.3$ and $287.3 \mathrm{eV}$ indicated the co-existence of $\mathrm{C}-\mathrm{C}, \mathrm{C}-\mathrm{O}, \mathrm{O}-\mathrm{C}-\mathrm{O}$ bonds and the peaks of $\mathrm{O} 1 \mathrm{~s}$ at 533.1 and $532.4 \mathrm{eV}$ also confirmed the existence of $\mathrm{C}-\mathrm{O}-\mathrm{C}$ and $\mathrm{C}-\mathrm{OH}$ bonds (Fig. S5). The binding energy of $\mathrm{C}=\mathrm{O}$ bond showed an increase from 288.1 to $288.7 \mathrm{eV}$ in $\mathrm{C} 1 \mathrm{~s}$ and a reduction from 531.7 to $531.5 \mathrm{eV}$ in $\mathrm{O} 1 \mathrm{~s}$, which implied $\mathrm{C}=\mathrm{O}$ as the main coordination binding mode with Pd NPs. These results revealed that metal Pd had been chemically confined in the CNF-Pd.

The morphology and size distribution of Pd NPs confined in CNF-Pd were further observed by TEM and SEM. The TEM image in Fig. 2a shows even-dispersed torispherical Pd NPs on the CNF substrate. Because the observation of Pd NPs did not need a phosphotungstic acid staining treatment, the non-conducting CNFs were hardly to distinguish. The sizes of Pd NPs measured from a high resolution TEM (HRTEM) image (Fig. 2b) ranged from 3 to $8 \mathrm{~nm}$, and the average diameter was $6.2 \mathrm{~nm}$ (Fig. 2d), close to that calculated from XRD by using the Scherrer equation $(6.4 \mathrm{~nm})$. The lattice fringe spacing was measured as $0.23 \mathrm{~nm}$ from Fig. 2c, also consistent with the $\operatorname{Pd}(111)$ lattice spacing of face centered cubic struc- ture [45].

The microstructure of CNF-Pd was 3D cellular-like at low magnification (Fig. 3a). With the magnification increase, it was found that the CNF-Pd foam consisted of CNF-based nanosheets, which were constituted by the interlaced CNFs (Fig. 3b and inset). Massive mesopores were formed within the CNFs and evenly distributed throughout the nanosheets (Fig. 3c). The EDS mapping of $\mathrm{C}, \mathrm{O}$, and $\mathrm{Pd}$ elements proved that Pd NPs were also evenly distributed across the whole nanosheets (Fig. 3d-f and Fig. S6). The ICP analysis gave a high Pd content of $9.6 \mathrm{wt} \%$ for the CNF-Pd. The specific surface area of the CNF-Pd was $20.9 \mathrm{~m}^{2} \mathrm{~g}^{-1}$ by the BET method, with a peak pore diameter of $5 \mathrm{~nm}$ (Fig. S7). Such a porous nanostructure was conducive to improving the fluid accessibility and providing sufficient active sites, and it therefore can enhance the reaction efficiency within the nanodomains $[27,46]$.

Catalytic hydrogenation of 4-nitrophenol was used as a model reaction to evaluate the catalytic activity of CNFPd. As shown in Fig. 4a, the absorption peak at $400 \mathrm{~nm}$ belongs to 4-nitrophenolate ion with the addition of $\mathrm{NaBH}_{4}$ in aqueous solution. Fig. $4 \mathrm{~b}$ shows the UV-Vis absorption spectra of the reaction mixtures correspond-
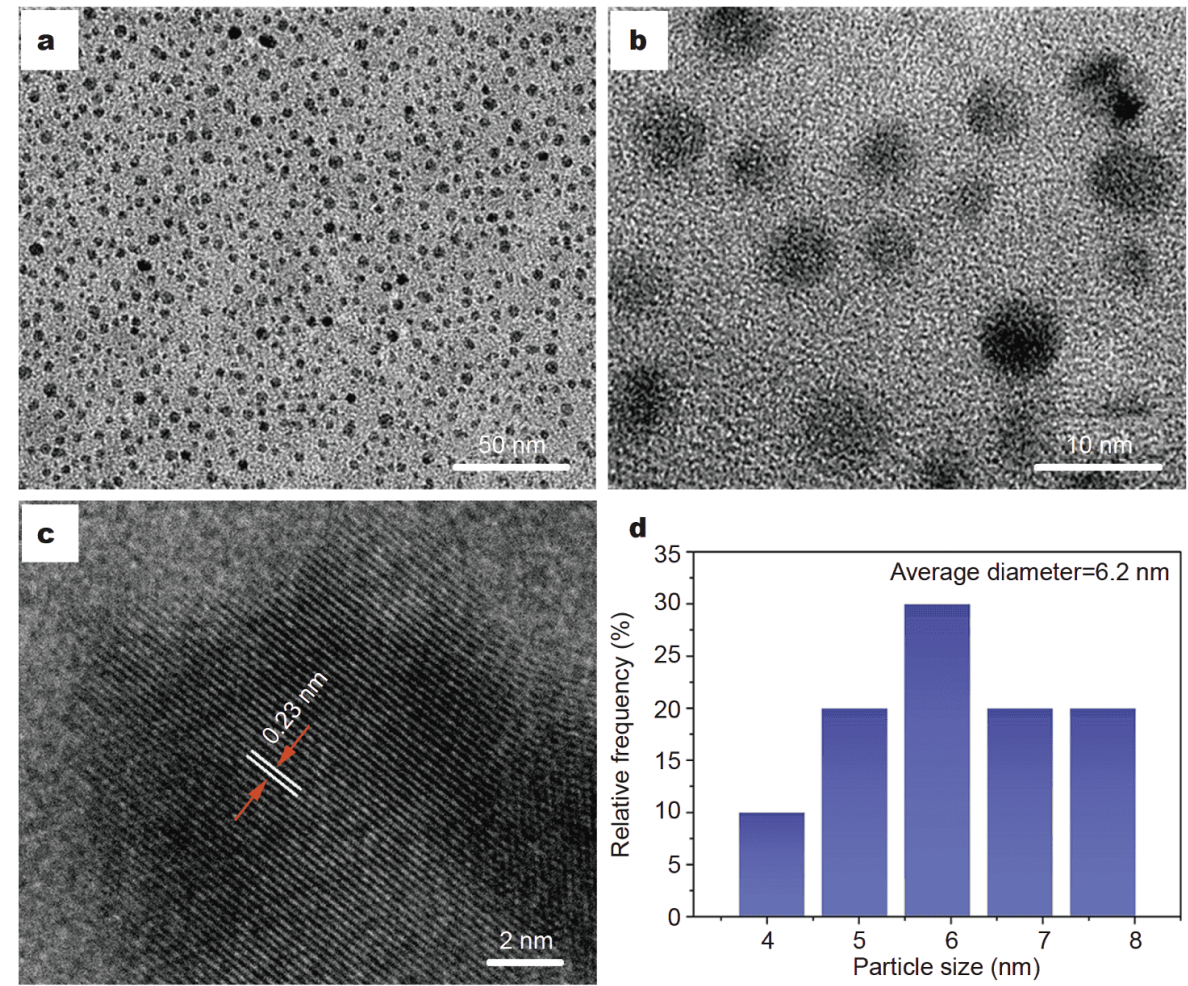

Figure 2 Morphology of the synthesized Pd NPs on CNFs: (a-c) TEM images and (d) size distribution of the Pd NPs. 

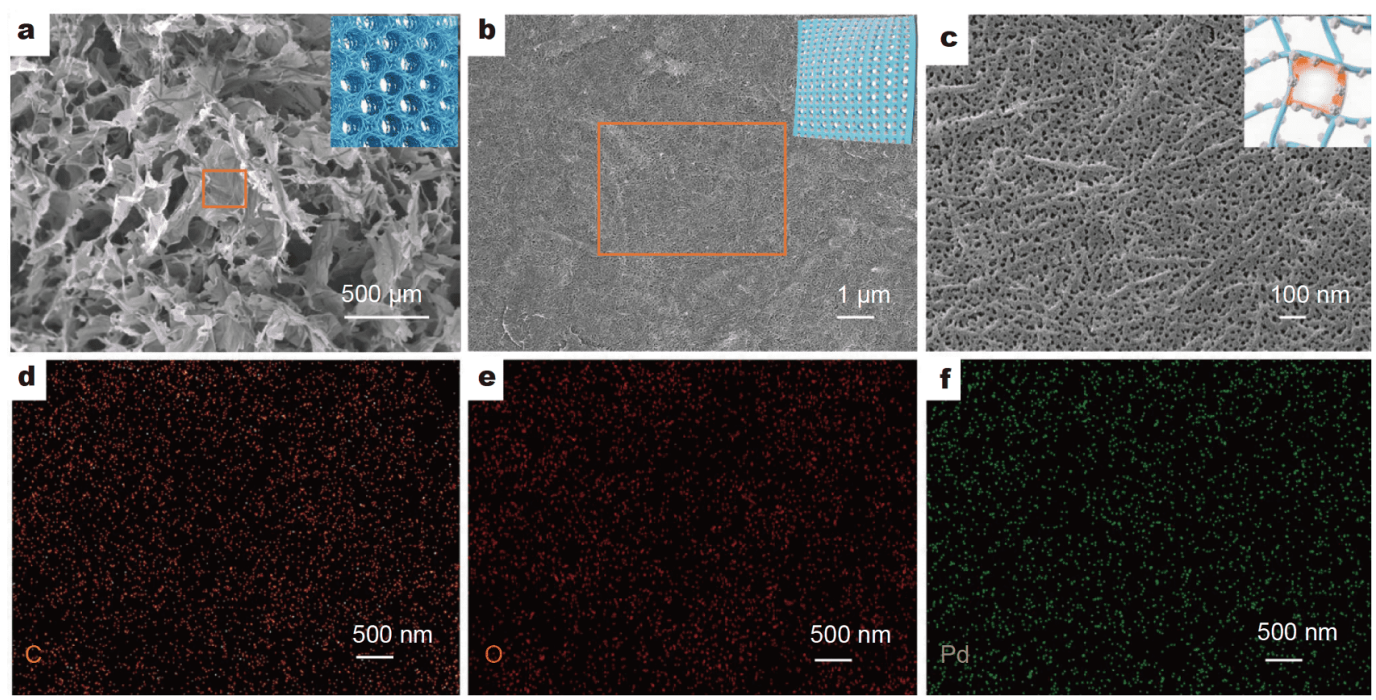

Figure 3 (a) SEM images of the CNF-Pd at (a, b) low magnification and (c) high magnification. The insets are diagrams of the CNF-Pd at different scales. (d-f) EDS mapping images of $\mathrm{C}$, $\mathrm{O}$ and Pd elements.
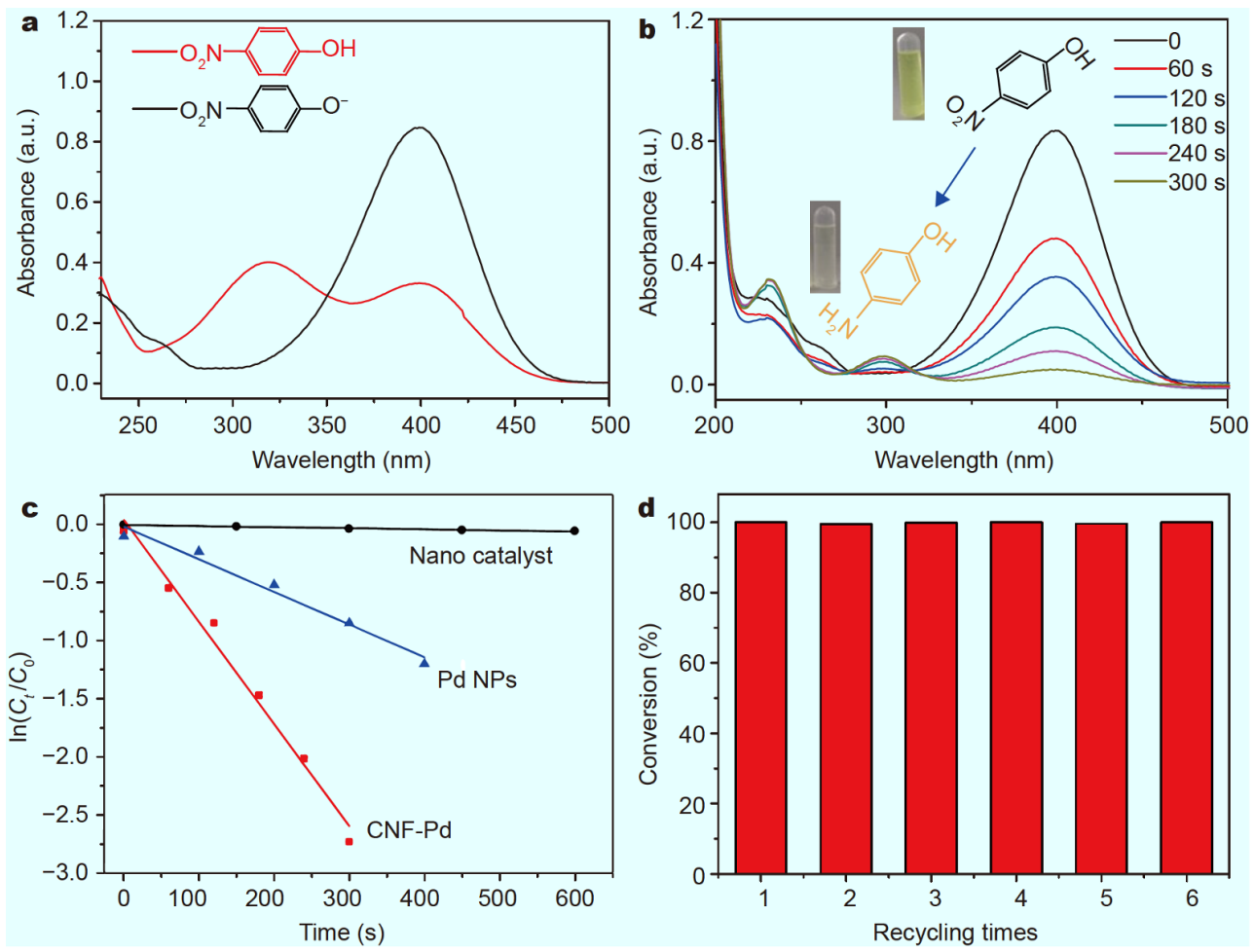

Figure 4 Use of CNF-Pd in the hydrogenation of 4-nitrophenol to 4-aminophenol at room temperature: (a) UV-Vis absorption spectra of 4nitrophenolate ions with (red curve) or without (black curve) $\mathrm{NaBH}_{4}$ in the aqueous solution; (b) UV-Vis absorption spectra of the reagents at different reaction times (insets show the photos of the color change before and after the reaction with the corresponding molecular structures); (c) $\ln \left(C_{t} / C_{0}\right)$ versus reaction time of CNF-Pd and Pd NPs (without CNF confinement) as a catalyst, respectively; (d) recycle uses of CNF-Pd for six successive cycles.

ing to different reaction times. The absorption peak at $400 \mathrm{~nm}$ gradually decreased with the reaction time. A new peak at $300 \mathrm{~nm}$ corresponding to 4 -aminophenol emerged and its intensity also increased with the reaction time. The $k$ of CNF-Pd was $8.8 \times 10^{-3} \mathrm{~s}^{-1}$, apparently higher than $2.82 \times 10^{-3} \mathrm{~s}^{-1}$ of the Pd NPs without CNF 
Table 2 The catalytic activity of CNF-Pd compared with other matrix-supported Pd catalysts in literatures for the catalytic reduction of 4-nitrophenol

\begin{tabular}{|c|c|c|c|c|c|}
\hline Entry & Sample & Pd loading (wt $\%)$ & $k\left(\times 10^{-3} \mathrm{~s}^{-1}\right)$ & TOF $\left(h^{-1}\right)$ & Ref. \\
\hline 1 & CNF-Pd & 9.6 & 8.8 & 2640 & This work \\
\hline 2 & Pd NPs & - & 2.8 & 436 & Control \\
\hline 3 & $\mathrm{Pd} /$ chitosan & 15 & 4.1 & - & {$[27]$} \\
\hline 4 & $\mathrm{Pd} @ P A N I-C S-\mathrm{Fe}_{3} \mathrm{O}_{4}$ & 3.5 & 3.7 & 1994 & {$[48]$} \\
\hline 5 & $\mathrm{Pd}-\mathrm{NiO}$ & 4.85 & 4.8 & - & {$[49]$} \\
\hline 6 & $\mathrm{Pd} / \mathrm{CeO}_{2}$ & 0.2 & 8.0 & 1068 & {$[50]$} \\
\hline 7 & $\mathrm{Pd} / \mathrm{GO}$ & 7.5 & 4.5 & - & {$[51]$} \\
\hline 8 & Pd-CNT & 9.8 & 6.2 & 810 & {$[52]$} \\
\hline 9 & $\mathrm{Pd}-\mathrm{C} / \mathrm{N}$ & 0.52 & 7.0 & 2520 & [53] \\
\hline 10 & Pd@MIL & 1.57 & 6.5 & 2088 & {$[54]$} \\
\hline 11 & Pd@VP2 & 7.7 & 3.7 & 1980 & [55] \\
\hline
\end{tabular}

confinement (Fig. 4c). The TOF value of CNF-Pd was $2640 \mathrm{~h}^{-1}$, six times higher than that of Pd NPs without CNF confinement $\left(436 \mathrm{~h}^{-1}\right)$ (Table S1). When comparing the TOF and $k$ values of CNF-Pd with other matrixsupported Pd catalysts (Table 2), the CNF-Pd showed higher catalytic efficiency than most of Pd catalysts with chitosan and carbon substrates, and comparable catalytic efficiency to those of Pd catalysts supported on metal oxide substrates. Such superior catalytic activity of CNF$\mathrm{Pd}$ could be ascribed to its abundant nanopores within the 3D structure and dense spatial confinement of Pd NPs on hydrophilic substrates. Therefore, the CNF-Pd could be reused at least six successive cycles without losing activity (Fig. 4d), showing good recyclability.

A possible explanation for the high catalytic performance of CNF-Pd was proposed. The catalytic mechanism of the hydrogenation of 4-nitrophenol involves the electron transfer from the $\mathrm{BH}_{4}^{-}$donor to the 4nitrophenol acceptor following the Langmuir-HinshelWood mechanism [47]. $\mathrm{NaBH}_{4}$ has a high theoretical hydrogen capacity, and $\mathrm{H}_{2}$ can be released under alcohol/ water condition. The CNF-Pd has a 3D nanoporous structure with abundant active catalytic sites which can generate spatial nano-confinement effect on the 4nitrophenol. In the nanopores of CNF-Pd, the increased effective concentration of 4-nitrophenolate ions and more $\mathrm{H}-\mathrm{Pd}$ bonds were formed by attaching to CNF-Pd surfaces, enhancing the catalytic activity. After the electron transfer finished, $\mathrm{H}^{+}$and 4-aminophenolate ions rearranged and 4-aminophenol desorbed from the CNF-Pd surfaces. These results suggest that the confinement of $\mathrm{Pd}$ within CNF matrices not only enhanced the catalytic activity but also improved the catalyst stability.

NS was used as a model compound to investigate the chemoselective activity of the CNF-Pd. Scheme 2 shows two possible hydrogenation reaction routes of NS [56]. One route is selective hydrogenation of the nitro group in NS to give AS; the other is selective hydrogenation of double bond in NS to generate ENB. Regardless of which route is initially followed, excessive hydrogenation of the products eventually produces 3-ethylaniline (EA).

Both solvent and reaction time were investigated for chemoselective conversion of NS with $\mathrm{NaBH}_{4}$ and CNF$\mathrm{Pd}$. It was found that the catalytic activity order of the solvent is methyl alcohol $(\mathrm{MeOH})>$ water $>$ ethanol $(\mathrm{EtOH})>$ ethylene glycol (MEG) (Table 3). The use of MEG or EtOH as solvent with a reaction time of $3 \mathrm{~h}$ could produce ENB with excellent selectivity of $100 \%$, but the conversion was relatively low (Table 3 , entries 1,2 ). The final hydrogenation product EA was obtained with $\mathrm{MeOH}$ or water as solvent and a reaction time of $3 \mathrm{~h}$, and the conversion reached $100 \%$ (entries 3, 4). These results indicate that the solvent played an important role in the selectivity of catalytic route [57]. Chemoselective conversion of NS to AS could be obtained with $80 \%-98 \%$ yield by choosing water, $\mathrm{MeOH}$, or water/MeOH $(1: 1$,

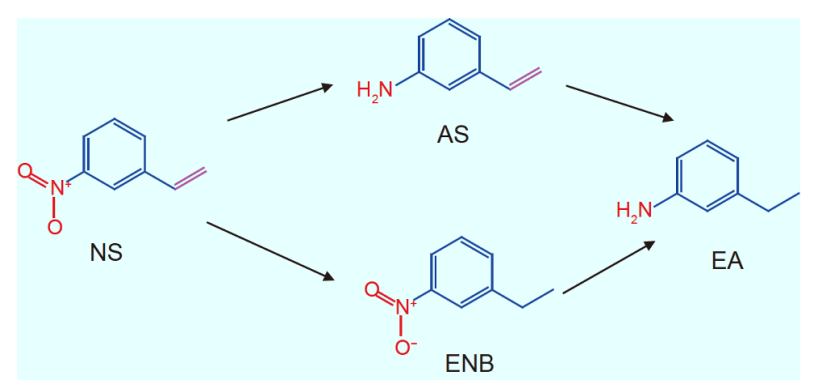

Scheme 2 Chemoselective conversion of NS to AS or ENB, and eventually EA. 
Table 3 Chemoselective conversion of NS with $\mathrm{NaBH}_{4}$ and CNF-Pd under different reaction conditions ${ }^{\mathrm{a}}$

\begin{tabular}{|c|c|c|c|c|c|c|c|}
\hline \multirow{2}{*}{ No. } & \multirow{2}{*}{ Catalyst } & \multirow{2}{*}{ Solvent $(10 \mathrm{~mL})$} & \multirow{2}{*}{ Time (h) } & \multirow{2}{*}{ Conversion of NS (\%) } & \multicolumn{3}{|c|}{ Selectivity (\%) } \\
\hline & & & & & AS & ENB & EA \\
\hline 1 & & MEG & 3 & 13 & 0 & 100 & 0 \\
\hline 2 & & $\mathrm{EtOH}$ & 3 & 50 & 0 & 100 & 0 \\
\hline 3 & & $\mathrm{MeOH}$ & 3 & 100 & 0 & 0 & 100 \\
\hline 4 & CNF-Pd/NaBH 4 & Water & 3 & 100 & 0 & 0 & 100 \\
\hline 5 & & $\mathrm{MeOH}$ & 1 & 100 & 96 & 0 & 4 \\
\hline 6 & & Water & 1 & 80 & 100 & 0 & 0 \\
\hline 7 & & Water/MeOH ${ }^{\mathrm{b}}$ & 1 & 98 & 100 & 0 & 0 \\
\hline
\end{tabular}

a) Reaction conditions: NS (0.5 mmol), CNF-Pd $(5 \mathrm{mg}), \mathrm{NaBH}_{4}(2 \mathrm{mmol})$, room temperature; b) volume ratio of water and $\mathrm{MeOH}$ is $1: 1$.

$v / v)$ as the solvent with a reaction time of $1 \mathrm{~h}$ (entries 57). Therefore, the combination of CNF-Pd and certain solvent can generate a chemoselectivity on the products. This is probably because protic solvents with higher hydrogen donating ability can help hydrogen transfer on the Pd NPs through the hydrogen spillover mechanism [5860].

\section{CONCLUSION}

In summary, a highly active and recyclable CNF-Pd catalyst was fabricated by a facile and green route. Ultrafine Pd NPs with an average diameter of $6.2 \mathrm{~nm}$ were spatially confined in the CNF matrices, with a high loading of $9.6 \mathrm{wt} \%$. The CNF-Pd showed high room-temperature catalytic activity in the hydrogenation of 4-nitrophenol by $\mathrm{NaBH}_{4}$. The reaction rate constant was $8.8 \times 10^{-3} \mathrm{~s}^{-1}$, and the TOF value reached $2640 \mathrm{~h}^{-1}$, which were far higher than those of Pd NPs without CNF confinement. The CNF-Pd was reusable even after six successive cycles due to the chemical immobilization of Pd NPs. The combination of CNF-Pd and suitable solvent could exhibit good chemoselectivity in the conversion of NS. Overall, we demonstrate a facile strategy to prepare recyclable Pd NPs by CNF confinement with enhanced catalytic activity and chemoselectivity.

Received 27 May 2020; accepted 23 June 2020; published online 24 September 2020

1 Balanta A, Godard C, Claver C. Pd nanoparticles for C-C coupling reactions. Chem Soc Rev, 2011, 40: 4973-4985

2 Dusselier M, Van Wouwe P, Dewaele A, et al. Lactic acid as a platform chemical in the biobased economy: The role of chemocatalysis. Energy Environ Sci, 2013, 6: 1415-1442

3 Bianchini C, Shen PK. Palladium-based electrocatalysts for alcohol oxidation in half cells and in direct alcohol fuel cells. Chem Rev, 2009, 109: 4183-4206

4 Lei Y, Wang Q, Peng S, et al. Electrospun inorganic nanofibers for oxygen electrocatalysis: Design, fabrication and progress. Adv
Energy Mater, 2020, doi: 10.1002/aenm.201902115

5 Barakat T, Rooke JC, Tidahy HL, et al. Noble-metal-based catalysts supported on zeolites and macro-mesoporous metal oxide supports for the total oxidation of volatile organic compounds. ChemSusChem, 2011, 4: 1420-1430

6 Kambe N, Iwasaki T, Terao J. Pd-catalyzed cross-coupling reactions of alkyl halides. Chem Soc Rev, 2011, 40: 4937-4947

7 Wang $\mathrm{D}$, Weinstein $\mathrm{AB}$, White $\mathrm{PB}$, et al. Ligand-promoted palladium-catalyzed aerobic oxidation reactions. Chem Rev, 2017, 118: 2636-2679

8 An N, Ainembabazi D, Reid C, et al. Microwave-assisted decarbonylation of biomass-derived aldehydes using Pd-doped hydrotalcites. ChemSusChem, 2020, 13: 312-320

9 Zhang X, Qu Z, Jiang H, et al. Selective catalytic hydrogenation of phenol to cyclohexanone over Pd@CN: Role of CN precursor separation mode. Can J Chem Eng, 2018, 97: 1506-1514

10 Mitra J, Zhou X, Rauchfuss T. Pd/C-catalyzed reactions of HMF: Decarbonylation, hydrogenation, and hydrogenolysis. Green Chem, 2015, 17: 307-313

11 Biffis A, Centomo P, Del Zotto A, et al. Pd metal catalysts for cross-couplings and related reactions in the 21st century: A critical review. Chem Rev, 2018, 118: 2249-2295

12 Liu L, Corma A. Metal catalysts for heterogeneous catalysis: From single atoms to nanoclusters and nanoparticles. Chem Rev, 2018, 118: 4981-5079

13 Lin Y, Qiao Y, Wang Y, et al. Self-assembled laminated nanoribbon-directed synthesis of noble metallic nanoparticle-decorated silica nanotubes and their catalytic applications. J Mater Chem, 2012, 22: 18314-18320

14 Le X, Dong Z, Liu Y, et al. Palladium nanoparticles immobilized on core-shell magnetic fibers as a highly efficient and recyclable heterogeneous catalyst for the reduction of 4-nitrophenol and Suzuki coupling reactions. J Mater Chem A, 2014, 2: 19696-19706

15 Zheng S, Yang P, Zhang F, et al. Pd nanoparticles encaged within amine-functionalized metal-organic frameworks: Catalytic activity and reaction mechanism in the hydrogenation of 2,3,5-trimethylbenzoquinone. Chem Eng J, 2017, 328: 977-987

16 Yang F, Ding S, Song H, et al. Single-atom Pd dispersed on nanoscale anatase $\mathrm{TiO}_{2}$ for the selective hydrogenation of phenylacetylene. Sci China Mater, 2020, 63: 982-992

17 Yang L, Chen Z, Cui D, et al. Ultrafine palladium nanoparticles supported on 3D self-supported $\mathrm{Ni}$ foam for cathodic dechlorination of florfenicol. Chem Eng J, 2019, 359: 894-901

18 Roig-Sanchez S, Jungstedt E, Anton-Sales I, et al. Nanocellulose 
films with multiple functional nanoparticles in confined spatial distribution. Nanoscale Horiz, 2019, 4: 634-641

19 Shi Q, Wang Y, Wang Z, et al. Three-dimensional (3D) interconnected networks fabricated via in-situ growth of $\mathrm{N}$-doped graphene/carbon nanotubes on Co-containing carbon nanofibers for enhanced oxygen reduction. Nano Res, 2016, 9: 317-328

20 Ding TY, Zhao ZG, Ran MF, et al. Superior activity of Pd nanoparticles confined in carbon nanotubes for hydrogen production from formic acid decomposition at ambient temperature. J Colloid Interface Sci, 2019, 538: 474-480

21 Lei Y, Shi Q, Han C, et al. N-doped graphene grown on silk cocoon-derived interconnected carbon fibers for oxygen reduction reaction and photocatalytic hydrogen production. Nano Res, 2016, 9: $2498-2509$

22 Zhao X, Jin Y, Zhang F, et al. Catalytic hydrogenation of 2,3,5trimethylbenzoquinone over Pd nanoparticles confined in the cages of MIL-101(Cr). Chem Eng J, 2014, 239: 33-41

23 Bergström L. Wood-inspired engineering materials. Sci China Mater, 2018, 61: 1625-1626

24 Li Y, Xu L, Xu B, et al. Cellulose sponge supported palladium nanoparticles as recyclable cross-coupling catalysts. ACS Appl Mater Interfaces, 2017, 9: 17155-17162

25 Chen F, Gong AS, Zhu M, et al. Mesoporous, three-dimensional wood membrane decorated with nanoparticles for highly efficient water treatment. ACS Nano, 2017, 11: 4275-4282

26 Cai Z, Fu J, Du P, et al. Reduction of nitrobenzene in aqueous and soil phases using carboxymethyl cellulose stabilized zero-valent iron nanoparticles. Chem Eng J, 2018, 332: 227-236

27 Dhanavel S, Manivannan N, Mathivanan N, et al. Preparation and characterization of cross-linked chitosan/palladium nanocomposites for catalytic and antibacterial activity. J Mol Liquids, 2018, 257: $32-41$

28 Li X, Dong F, Zhang L, et al. Cellulosic protic ionic liquids hydrogel: A green and efficient catalyst carrier for Pd nanoparticles in reduction of 4-nitrophenol in water. Chem Eng J, 2019, 372: 516525

29 Zhao D, Zhu Y, Cheng W, et al. Cellulose-based flexible functional materials for emerging intelligent electronics. Adv Mater, 2020, doi: 10.1002/adma.202000619

30 Kaushik M, Moores A. Review: Nanocelluloses as versatile supports for metal nanoparticles and their applications in catalysis. Green Chem, 2016, 18: 622-637

31 Rezayat M, Blundell RK, Camp JE, et al. Green one-step synthesis of catalytically active palladium nanoparticles supported on cellulose nanocrystals. ACS Sustain Chem Eng, 2014, 2: 1241-1250

$32 \mathrm{Wu} \mathrm{X}$, Shi Z, Fu S, et al. Strategy for synthesizing porous cellulose nanocrystal supported metal nanocatalysts. ACS Sustain Chem Eng, 2016, 4: 5929-5935

33 An $\mathrm{X}$, Long $\mathrm{Y}, \mathrm{Ni} \mathrm{Y}$. Cellulose nanocrystal/hexadecyltrimethylammonium bromide/silver nanoparticle composite as a catalyst for reduction of 4-nitrophenol. Carbohydr Polyms, 2018, 156: $253-258$

34 Wang B, Dai L, Yang G, et al. A highly efficient thermo responsive palladium nanoparticles incorporated guar gum hydrogel for effective catalytic reactions. Carbohydr Polyms, 2019, 226: 115289

35 Zhang K, Shen M, Liu H, et al. Facile synthesis of palladium and gold nanoparticles by using dialdehyde nanocellulose as template and reducing agent. Carbohydr Polyms, 2018, 186: 132-139

36 Jebali Z, Granados A, Nabili A, et al. Cationic cellulose nanofibrils as a green support of palladium nanoparticles: catalyst evaluation in Suzuki reactions. Cellulose, 2018, 25: 6963-6975

37 Liu Y, Chen W, Xia Q, et al. Efficient cleavage of lignin-carbohydrate complexes and ultrafast extraction of lignin oligomers from wood biomass by microwave-assisted treatment with deep eutectic solvent. ChemSusChem, 2017, 10: 1692-1700

38 Sirviö JA, Visanko M. Anionic wood nanofibers produced from unbleached mechanical pulp by highly efficient chemical modification. J Mater Chem A, 2017, 5: 21828-21835

39 Liu Y, Guo B, Xia Q, et al. Efficient cleavage of strong hydrogen bonds in cotton by deep eutectic solvents and facile fabrication of cellulose nanocrystals in high yields. ACS Sustain Chem Eng, 2017, 5: 7623-7631

40 Ma Y, Xia Q, Liu Y, et al. Production of nanocellulose using hydrated deep eutectic solvent combined with ultrasonic treatment. ACS Omega, 2019, 4: 8539-8547

41 Chen X, Wu G, Chen J, et al. Synthesis of "clean" and welldispersive Pd nanoparticles with excellent electrocatalytic property on graphene oxide. J Am Chem Soc, 2011, 133: 3693-3695

42 Movahed SK, Dabiri M, Bazgir A. Palladium nanoparticle decorated high nitrogen-doped graphene with high catalytic activity for Suzuki-Miyaura and Ullmann-type coupling reactions in aqueous media. Appl Catal A-Gen, 2014, 488: 265-274

43 Zhao Z, Zhang J, Wang Y, et al. Thermally stable Pd/reduced graphene oxide aerogel catalysts for solvent-free oxidation of benzyl alcohol. Chem Phys Lett, 2020, 746: 137306

44 Tian M, Long Y, Xu D, et al. Hollow mesoporous silica nanotubes modified with palladium nanoparticles for environmental catalytic applications. J Colloid Interface Sci, 2018, 521: 132-140

45 Dong B, Pei Y, Zhao F, et al. In situ quantitative single-molecule study of dynamic catalytic processes in nanoconfinement. Nat Catal, 2018, 1: 135-140

46 Patra S, Naik AN, Pandey AK, et al. Silver nanoparticles stabilized in porous polymer support: A highly active catalytic nanoreactor. Appl Catal A-Gen, 2016, 524: 214-222

47 Wunder S, Polzer F, Lu Y, et al. Kinetic analysis of catalytic reduction of 4-nitrophenol by metallic nanoparticles immobilized in spherical polyelectrolyte brushes. J Phys Chem C, 2010, 114: 88148820

48 Ayad MM, Amer WA, Kotp MG. Magnetic polyaniline-chitosan nanocomposite decorated with palladium nanoparticles for enhanced catalytic reduction of 4-nitrophenol. Mol Catal, 2017, 439: $72-80$

49 Kaur N, Sood A, Bhasin KK, et al. Metallosurfactants derived $\mathrm{Pd}-\mathrm{NiO}$ nanocomposite for remediation of nitrophenol in water. J Mol Liquids, 2019, 288: 111018

50 Liu B, Yu S, Wang Q, et al. Hollow mesoporous ceria nanoreactors with enhanced activity and stability for catalytic application. Chem Commun, 2013, 49: 3757-3759

51 Bramhaiah K, John NS. Hybrid films of reduced graphene oxide with noble metal nanoparticles generated at a liquid/liquid interface for applications in catalysis. RSC Adv, 2013, 3: 7765-7773

52 Kim JD, Choi MY, Choi HC. Catalyst activity of carbon nanotube supported Pd catalysts for the hydrogenation of nitroarenes. Mater Chem Phys, 2016, 173: 404-411

53 Long Y, Liu Y, Zhao Z, et al. Distinctive morphology effects of porous-spherical/yolk-shell/hollow Pd-nitrogen-doped-carbon spheres catalyst for catalytic reduction of 4-nitrophenol. J Colloid Interface Sci, 2017, 496: 465-473

$54 \mathrm{Xu}$ B, Li X, Chen Z, et al. Pd@MIL-100(Fe) composite nanoparticles as efficient catalyst for reduction of 2/3/4-nitrophenol: 
Synergistic effect between Pd and MIL-100(Fe). Microporous Mesoporous Mater, 2018, 255: 1-6

55 Zhu J, Lu X, Li Y, et al. A rotavirus virus-like particle confined palladium nanoreactor and its immobilization on graphene oxide for catalysis. Catal Lett, 2020, doi: 10.1007/s10562-020-03252-6

56 Zhang S, Chang CR, Huang ZQ, et al. High catalytic activity and chemoselectivity of sub-nanometric Pd clusters on porous nanorods of $\mathrm{CeO}_{2}$ for hydrogenation of nitroarenes. J Am Chem Soc, 2016, 138: 2629-2637

57 Boronat M, Concepción P, Corma A, et al. A molecular mechanism for the chemoselective hydrogenation of substituted nitroaromatics with nanoparticles of gold on $\mathrm{TiO}_{2}$ catalysts: A cooperative effect between gold and the support. J Am Chem Soc, 2007, 129: 16230-16237

58 Merte LR, Peng G, Bechstein R, et al. Water-mediated proton hopping on an iron oxide surface. Science, 2012, 336: 889-893

59 Zhao D, Zhu Y, Cheng W, et al. A dynamic gel with reversible and tunable topological networks and performances. Matter, 2020, 2: 390-403

60 Wei X, Zhou M, Zhang X, et al. Amphiphilic mesoporous sandwich-structured catalysts for selective hydrogenation of 4-nitrostyrene in water. ACS Appl Mater Interfaces, 2019, 11: 3911639124

Acknowledgements This work was supported by the National Natural Science Foundation of China (31925028 and 31670583), and the Special Project for Double First-Class-Cultivation of Innovative Talents (000/ 41113102).

Author contributions $\mathrm{Yu} \mathrm{H}$ and Zhang $\mathrm{X}$ supervised the project; Meng J, Liu Y and Shi X performed the experiments; Meng J, Liu Y and $\mathrm{Yu} \mathrm{H}$ wrote the paper with supports from Chen $\mathrm{W}$ and Zhang X. All authors contributed to the general discussion.

Conflict of interest The authors declare that they have no conflict of interest.

Supplementary information online version of the paper.

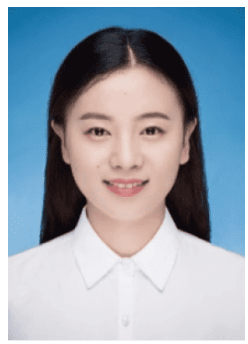

Juan Meng is currently a $\mathrm{PhD}$ candidate at Northeast Forestry University, China. She received her BSc degree in wood science and engineering from Anhui Agricultural University in 2016. Her research interest focuses on the design and synthesis of biomass-based nanomaterials for catalytic applications.

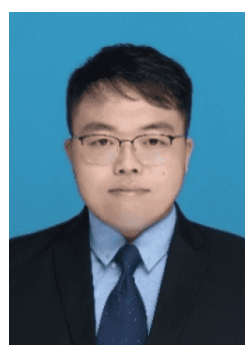

Yongzhuang Liu received his $\mathrm{PhD}$ degree in 2019 from Northeast Forestry University, China. He was a visiting student at the University of Groningen, the Netherlands during 2018-2019. Now he is an associate professor at the Key Laboratory of Bio-based Material Science and Technology of Ministry of Education, Northeast Forestry University. His research interest lies in the valorization of lignocellulosic renewable materials using green solvents.

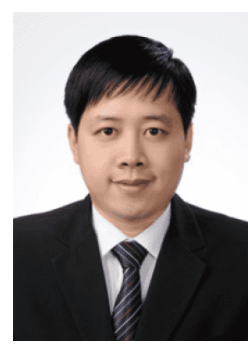

Haipeng Yu received his $\mathrm{PhD}$ degree in 2005 from Northeast Forestry University, China. He is currently a full professor at the Key Laboratory of Bio-based Material Science and Technology of Ministry of Education, Northeast Forestry University. His research interest focuses on the green conversion of sustainable biomass for developing bio-based functional materials. Currently, he is interested in the cellulose and nanocellulosebased functional materials, including catalytic materials, energy storage materials and flexible intelligent electronics.

\section{纳米纤维素限域钯纳米颗粒的室温催化活性及化 学选择性}

孟娟 ${ }^{\dagger}$, 刘永壮 ${ }^{\dagger}$, 史晓超, 陈文帅, 张显权 ${ }^{*}$, 于海鹏 ${ }^{*}$

摘要 本文利用水合低共熔溶剂结合超声法制备了羧基化纤维素 纳米纤丝(CNF), 并利用其表面的羧基官能团为反应活性位点原位 合成了具有均匀分散性和高负载量 $(9.6 \mathrm{wt} \%$ )的CNF-Pd复合催化 剂. 研究结果表明: CNF-Pd具有显著的限域特性, 包括在CNF表面 羧基官能团上原位固定生长 Pd纳米颗粒的化学限域和 CNF冷冻干 燥过程中自组装形成的空间结构限域. CNF-Pd在4-硝基苯酚加氢 模型反应中表现出优异的室温催化活性, 其最高速率常数为 $8.8 \times 10^{-3} \mathrm{~s}^{-1}$, 最高催化活性的转化频率为 $2640 \mathrm{~h}^{-1}$. CNF-Pd催化剂 在水性介质中显示出良好的化学稳定性和可回收性, 至少重复使 用6个循环不会失活. 此外, CNF-Pd催化剂还可实现3-硝基苯乙烯 在醇/水共溶剂中向 3 -氨基苯乙烯的化学选择性加氢, 且具有 $80 \%$ 98\%的高选择性产率. 综合上述结果, CNF在构建具有高室温催化 活性及可回收利用的金属纳米颗粒催化剂方面具有广泛应用潜力 和发展空间. 